\title{
The Evolution and Growth of Nearby Galaxy Groups
}

Katiya Fosdick

\author{
University of Wisconsin - Madison
}

\begin{abstract}
:
This project strives to study the relationship between the group environment and individual galaxy properties. Group richness is used as a proxy for different environmental conditions and mass to form a sample of 40 low mass $(3 \leq N<10)$, 40 medium mass $(10 \leq N<20)$, and 40 high mass $(20 \leq N)$ groups from the Berlind et al. 2006 volume-limited Mr18 catalog and Tempel et al. 2014 flux-limited catalog. Unique color-magnitude-position and color-stellar mass-position diagrams are produced for each sample group. I identified dynamically active and inactive groups using the magnitude gap through a version of methodology described in Raouf et al. 2019. Project figures are used to analyze how BGG properties, dynamic activity, and group appearance depend on group richness. Analysis focuses on BGGs to understand how one evolutionary mechanism, mergers, is impacted by basic differences in a group's environment.
\end{abstract}

\section{INTRODUCTION:}

Like other matter in the universe, galaxies gather hierarchically in gravitationally bound structures that are larger than themselves (White \& Rees 1978). One of these structures is galaxy groups, which themselves are fundamental substructures of clusters and superclusters. Galaxy groups contain 3-50 gravitationally-bound galaxies and hot or warm gas that exists between them, known as the intragroup medium (IGrM). Numerous studies confirm that most galaxies in the local universe $(z<1)$ reside and evolve in galaxy groups (Geller \& Huchra 1983, Tully 1987, Eke et al. 2004, Yang et al. 2007, Tago et al. 2008). Galaxy groups continue to demonstrate their utility for studying galaxy evolution in the local universe.

The evolution of galaxies in groups is closely related to their local environment. The group environment is the unique local condition in a galaxy group produced by the members of the group and the IGrM, though it is defined differently in many studies. Galaxy-Galaxy interactions and Galaxy-IGrM interactions occur at higher rates in dense environments, acting to drive the evolution of galaxies in groups (Dressler 1984, Just et al. 2010, Freeland et al. 2009). The IGrM and member position, density, and velocity dispersion determine which mechanisms are most important in driving the evolution of a galaxy (Just et al. 2010). Ram pressure stripping, starvation and tidal interactions act on satellite galaxies, while mergers or accretion form brightest group galaxies (BGGs): dormant, early-type galaxies that are the most luminous and massive members of their group (Shen et al. 2014, Mahtessian et al. 2017). These mechanisms quench galaxy gas content to produce red, quitescent, E/S0-dominated galaxy populations in groups and alter the group environment (Dressler 1980, Cooray 2005). Member galaxy properties such as color, morphology, and star formation rate (SFR) are altered by evolutionary processes and correlated with the group environment, though the specifics of this complex relationship are poorly understood (Helsdon \& Ponman 2003, Freeland et al. 2009, Tempel et. al. 2017). By using group richness as a proxy for different environmental conditions, this project and Fosdick 2020 explore how individual galaxy properties depend on the richness of their host group.

Like other members of galaxy groups, BGG properties are influenced by the group environment (Shen et al. 2014, Luparello et al. 2015). The magnitude gap $\Delta M_{r_{12}}$ is the difference in r-band absolute magnitude between the 1st and 2nd brightest galaxies. When combined with the 
magnitude gap, BGGs can be used to identify historical or current evidence of mergers: galaxygalaxy interactions that cause starbursts and increased SFR in a galaxy group's central galaxy. The magnitude gap may be a crude indicator of a group's dynamic age, the amount of time a group has been dynamically active. The magnitude gap is interpreted through BGGs. A larger magnitude gap generally indicates more mergers have occurred to increase the mass of the BGG and that the group has been dynamically active for longer. A large magnitude gap may indicate accretion onto the BGG has stopped. BGGs in unrelaxed groups are bluer at specific wavelengths and have higher star formation rates (SFR) than those in relaxed groups, indicative of environments with frequent mergers (Raouf et al. 2019). This project uses the properties of BGGs, unrelaxed, and relaxed groups to study the effects of group richness on mergers.

\section{SAMPLE:}

This project uses a sample of 120 galaxy groups separated into three mass categories: high mass groups ( $N \geq 20$ members), medium mass groups ( $10 \leq N<20$ members), and low mass groups ( $3 \leq N<10$ members). Twenty groups in each richness category are selected from two galaxy catalogs separately to produce a 60 group sample from each catalog. One of these galaxy catalogs is the Mr18 group catalog described in Berlind et al. 2006. Berlind et al. 2006 produced this volume-limited catalog using a friends-of-friends algorithm from galaxies in the 3rd SDSS data release (DR3), with a limiting magnitude $M_{r}=-18$ (Berlind et al. 2006). I also obtained sample groups from the Tempel et al. 2014 flux-limited catalog, containing member galaxies from the 10th SDSS data release (DR10) and a modified friends-of-friends algorithm. While the Tempel et al. 2014 flux-limited catalog is complete below $M_{r}=-18$, its limiting magnitude of $M_{r}=17.77$ allows for the observation of dimmer galaxies than those in the Berlind et al. 2006 volume-limited catalog. These catalogs provide complete position, redshift (z), color (g-r), and $M_{r}$ data for each member galaxy. Overall, the project sample contains 1812 galaxies. A table listing all groups in the project sample is given in Supplemental Files.

Both catalogs have effects that impact the sample data. To account for $M_{r}$, g-r, and z data lost through fiber collisions or survey edge effects, Berlind et al. 2006 replaced missing data with that of a galaxy's nearest neighbor in the group. However, this correction creates repeat galaxies, affecting $4 \%$ of the project sample, causing a slight bias in project g-r and $M_{r}$ data. The Tempel et al. 2014 group eliminated member galaxies with extraneous colors from their catalog. I removed three Tempel et al. 2014 galaxies with outlying color data from the project sample. This correction caused the magnitude gap to be incalculable for one group. Generally, since SDSS data releases are cumulative, I expect Tempel et al. 2014 groups to be more complete than Berlin et al. 2006 groups. Data incompleteness is an issue in the project sample. 82\% of project galaxies have log stellar masses returned by SDSS. This data is complete for $70 \%$ of BGGs. Morphologies are known for $60 \%$ of sample galaxies and $70 \%$ of BGGs.

\section{METHODS:}

I used the following computational and graphical methods during this project. I include amendments to methods described in detail in Fosdick 2020 under their Fosdick 2020 headings. I used methods listed under "Figures" in Fosdick 2020 to produce the project's 240 colormagnitude-position and color-stellar mass-position diagrams. 
SDSS Queries: I made SDSS queries using SDSS DR8 ObjIDs from the Tempel et al. 2014 catalog and DR8 ObjIDs assigned to Berlind et al. 2006 galaxies using SDSS SkyServers's CrossID tool. I queried SDSS DR16 for Conroy et al. 2009 best-fit log stellar masses for all sample galaxies. I sourced morphological classifications for Berlind et al. 2006 galaxies from Galaxy Zoo (Lintott et al. 2010), while I used in-catalog morphologies described in Tempel et al. 2011 for Tempel et al. 2014 galaxies.

Magnitude Gap, BGG, Relaxed/Unrelaxed groups: I calculated the magnitude gap, $\Delta M_{r_{12}}=M_{r_{2}}-M_{r_{1}}$ by subtracting the r-band absolute magnitude of a group's brightest galaxy from the r-band absolute magnitude of the 2 nd brightest galaxy. The BGG is the galaxy with the group's brightest $M_{r}$ value. I identified relaxed and unrelaxed groups using the magnitude gap criterion in Raouf et al. 2019. Raouf et al. 2019 identify groups with a high magnitude gap, $\Delta M_{r_{12}} \geq 1.7$, as dynamically inactive or relaxed while those with a low magnitude gap, $\Delta M_{r_{12}} \leq 0.5$ as dynamically active or unrelaxed. These classifications are corroborated by Dariush et al. 2010.

Stellar Mass Scaling: To scale galaxy markers by their stellar masses in Figures 1-240, I exponentiated SDSS best-fit stellar log masses by a base 10 to produce best-fit stellar masses for each galaxy. I multiplied member galaxy best-fit stellar masses by an arbitrary factor of $6 \times 10^{-9}$ to make them visible when plotted. I used group member best-fit stellar $\log$ masses to produce color-stellar mass-position diagrams. Best-fit stellar log mass data is incomplete for the project sample. As a result, some project sample galaxies are missing in these figures.

Magnitude Scaling: I replaced the arbitrary scaling factor of 20 in Fosdick 2020 with 25 in this project to fit the expanded project sample better.

\section{KEY RESULTS:}

The project's 240 figures (located in Supplemental Files) allow me to compare the group environment across an extensive range of conditions. Differences between the samples from each project catalog become evident in these figures. Generally, Tempel et al. 2014 galaxies are bluer than those in the Berlind et al. 2006 catalog. This effect is especially apparent in the high mass sample, but it is present in all richness categories. In Fosdick 2020, I noted a negative correlation between the r-band luminosity of member galaxies and their g-r spread color in the Berlind et al. 2006 high mass sample. As the luminosity of sample galaxies increased, the corresponding sample galaxy color became progressively redder with a smaller g-r range. I postulated in Fosdick 2020 that this trend was caused by a bias towards redness in the Berlind et al. 2006 catalog and that highly luminous galaxies with $\mathrm{g}-\mathrm{r}<1$ were missing from this sample. My hypothesis is corroborated by some high mass Tempel et al. 2014 groups. Tempel et al. 2014 introduces many galaxies with small r-band luminosities, small stellar masses, and relatively blue g-r colors $(g-r<0.4)$ to the project sample. This introduction does not suggest that limiting magnitude causes a red bias in the Berlind et al. 2006 groups, as both catalogs are complete to $M_{r}=-18$, and extreme reddening is not observed in Tempel et al. 2014 groups for large $M_{r}$ values. 
The most luminous galaxies in the sample are found in high richness groups. They are not always the reddest galaxies in the project sample, with g-r $\geq \sim 0.8$. Tempel et al. 2014 groups have bluer BGGs than Berlind et al. 2006 groups. Notably, the luminosity of BGGs seem to depend weakly on the richness of their host group. As the richness of my subsamples increases in Table 1, the r-band luminosity of a group's brightest members tends to brighten by about one magnitude. This general dependency suggests that member density, a property of the group environment, may impact the luminosities of BGGs. This result should be checked with more narrowly defined richness subsamples. This result indicates the importance of mergers in the formation of BGGs, as more massive groups allow more luminous and massive BGGs to form. While this has not been confirmed, Tempel et al. 2014 groups seem to contain fewer luminous galaxies than Berlind et al. 2006 groups of the same richness category. Differences in each catalog's completeness may cause this trend. Tempel et al. 2014 groups contain the most luminous galaxies in the sample. These findings may change if either catalog sample was isolated and observed for these trends independently, although my analysis suggests this dependency exists in both catalogs independently.

Like the luminosity of BGGs, the probability that a group is relaxed or unrelaxed seems to depend on its richness. In the low mass sample, $30 \%$ of galaxy groups are unrelaxed and $25 \%$ of galaxy groups are relaxed, according to their magnitude gap and criterion outlined by Raouf et al. 2019. In the medium mass sample, $48 \%$ of galaxy groups are unrelaxed and $8 \%$ are relaxed. In the high mass sample, $43 \%$ of groups are unrelaxed and 3\% are relaxed. Medium and high mass groups seem to host more dynamic activity in The Local Universe, suggesting relatively rich groups provide environments where galaxy evolution and group change are more likely to occur. The dynamic activity of a galaxy group seems to depend, at least weakly, on the group's richness. Future work will examine the implications this result has on group formation.

Similar characteristics of dynamically relaxed and unrelaxed groups of all richness categories are illustrated by Figures 1-12, a selection of the 240 color-magnitude-position and color-stellar mass-position diagrams produced for each group in the total project sample. Unrelaxed groups tend to manifest physically in two different ways. Class I occurs when two or three galaxies in an unrelaxed group have similar $\mathrm{M}_{\mathrm{r}}$ values that are noticeably larger than those of surrounding members. The $\mathrm{M}_{\mathrm{r}}$ values of these galaxies are similar enough that they appear to be "competing" for the title of BGG. The BGG designation may be evident, but the BGG is only slightly brighter than other luminous galaxies in the group. Class I unrelaxed galaxy groups are observed in Berlind et al. 2006 groups 82 (Fig. 2), and 120 and Tempel et al. 2014 groups 8, 39 (Fig. 1), and 49. Tempel et al. 2014 groups 115 (Fig. 7) and 221 are obvious Class I galaxy group examples. Nothing about the Tempel et al. 2014 catalog suggests a possibility for repeated galaxies in Tempel et al. 2014 groups 115 and 221. Class II occurs in unrelaxed galaxy groups where most group members have similar $M_{r}$ values and no notable $B G G$, or when 4-5+ members fit the criterion of Class I and compete for the BGG designation. Similar member galaxy g-r colors may be expected in Class II groups, though this requires further confirmation. Class II galaxy groups are observed in Berlind et al. 2006 groups 24 (Fig. 3), 71, 1412, and 3896 (Fig. 5), and Tempel et al. 2014 group 24 (Fig. 4). Notably, these selected groups have no fiber collisions or few fiber collisions that don't alter the defining characteristic of Class II galaxy groups. I found it easiest to identify Class I galaxy groups in low mass groups and Class II galaxy groups in high mass groups. I found examples of both classes in all richness categories. The identification of these 
effects is subjective and requires further development and codification. Some unrelaxed galaxy groups appear to present a melding of the two classes, such as Berlind et al. 2006 group 1039, 3064 (Fig. 6), and 9722. But the two defining features of Class I and Class II galaxy groups are obvious and differentiable when compared in their extremes. The designation of an unrelaxed galaxy group as Class I or Class II does not seem to depend on the group's magnitude gap.

Relaxed groups are characterized by more homogeneous properties than unrelaxed groups. Relaxed or Class III galaxy groups contain one obvious BGG with an r-band absolute magnitude $\Delta M_{r_{12}}$ larger than the 2 nd brightest member galaxy. Tempel et al. 2014 group 224 (Fig. 10) is an example of a Class III galaxy group. BBGs in Class III groups range in magnitude from $-21.625 \leq M_{r} \leq-19.090$. When log stellar-mass data is available, BGGs are the most massive members of Class III groups. Surprisingly, of the nine Class III BGGs with morphological classifications, $78 \%$ of these BGGs are spiral galaxies. BGGs in Class III groups are also relatively blue. With $g-r_{\min }=0.533,64 \%$ of relaxed BGGs have g-r $<0.8$. Tempel et al. 2014 groups $21,33,37$, and 40 have anomalously high magnitude gaps. Missing galaxies below the catalog's completeness threshold of $M_{r}=-18$ likely explain these outliers.

Intermediate Class IV groups with $0.5<\Delta M_{r_{12}}<1.7$ are neither relaxed nor unrelaxed. One BGG is usually identifiable in these groups, though other members have comparable $M_{r}$ values, as illustrated by Berlind et al. 2006 group 8245 (Fig. 9) or 1415.

BGGs in intermediate and unrelaxed groups throughout the sample tend to have larger stellar masses than their relaxed counterparts at comparable $\mathrm{M}_{\mathrm{r}}$ values, as demonstrated in Figures 7-12. This trend is discussed briefly in Fosdick 2020 and is seemingly unaffected by group richness or BGG color. Larger BGG log stellar masses in intermediate and unrelaxed groups may reflect differences in the galaxies' star formation histories or stellar populations.

\begin{tabular}{|l|l|l|l|l|}
\hline BGG $M_{r}$ & $-23<M_{r} \leq-22$ & $-22<M_{r} \leq-21$ & $-21<M_{r} \leq-20$ & $-20<M_{r} \leq-19$ \\
\hline Low Mass & & $18 \%$ & $40 \%$ & $43 \%$ \\
\hline Medium Mass & & $40 \%$ & $50 \%$ & $8 \%$ \\
\hline High Mass & $18 \%$ & $58 \%$ & $20 \%$ & \\
\hline
\end{tabular}

Table 1: Richness Category v. BGG r-band Absolute Magnitude Table

The percentage of BGGs in each absolute magnitude bin, $M_{r}$, contains the brightest BGGs in each mass sample.

Each project subsample has 40 galaxy groups. Some $M_{r}$ bins that contain BGGs are not included.

\section{DISCUSSION:}

My extension of Fosdick 2020's project sample further confirms that galaxies in groups are primarily red, as demonstrated by an abundance of positive g-r colors in the project sample. Upon comparison, the Tempel et al. 2014 catalog seems to confirm that g-r color data from the Berlind et al. 2006 catalog has a red bias. The Tempel et al. 2014 flux-limited catalog introduces luminous galaxies $\left(-22<M_{r}<-21\right)$ with g-r $\leq \sim 0.8-0.85$ that Fosdick 2020 hypothesized the Berlind et al. 2006 catalog was missing. Tempel et al. 2014 introduces blue galaxies (g-r $>0)$ and relatively blue galaxies $(\mathrm{g}-\mathrm{r}<0.4$ ) expected to form $\sim 10 \%$ of the galaxy population in high 
density environments (Dressler 1984). These galaxies were largely absent from the Berlind et al. 2006 sample. The project sample's $\mathrm{M}_{\mathrm{r}}=-18$ limiting magnitude and catalog incompleteness may disproportionately eliminate blue galaxies.

BGGs are an essential focus of this project. BGG luminosity seems to depend weakly on group richness. Since BGGs grow via the accretion of satellite galaxies in a group or cluster, it makes sense that groups with more mass will have more massive, brighter BGGs. The weak dependency of BGG luminosity on group richness may be affected by evolutionary mechanisms that operate on satellite galaxies. Additionally, the mass categories used in my analysis contain a broad range of $\mathrm{N}$ group members. A more apparent dependency between richness and BGG luminosity may become evident by examining this relationship through a function of $\mathrm{N}$ instead of broad mass categories.

Where morphological data is available, a surprising number of BGGs, especially in dynamically relaxed groups, are spirals. Generally, spiral galaxies contain high neutral atomic and molecular gas content uncharacteristic of multiple mergers. Mergers are expected to exhaust the galaxies' atomic and molecular gas content. BGGs are typically S0 galaxies with red g-r colors, low SFR, and depleted gas content. S0 galaxies are usually found in intermediate-density environments, potentially explaining the frequency of spiral BGGs in relaxed groups. Most relaxed groups are found in the low mass project subsample, which contains groups that are richer than the field, but less rich than high or medium mass groups (Dressler 1984). Raouf et al. 2019 found a higher concentration of early-type galaxies in relaxed groups, indicating environmental effects could cause my results, though Mahtessian et al. 2017 corroborate that I expect more spiral BGGs at $\Delta M_{r_{12}}>1.5$.

Group dynamic activity appears to be related to group richness. The number of relaxed Class III groups in my project sample drops off sharply with increased richness. The fraction of unrelaxed groups increases with richness, though I need to establish a more evident dependency on $\mathrm{N}$ group members. Project data suggests that high mass groups are the most dynamically active, corroborating current knowledge that the frequency of evolutionary mechanisms increase in denser groups. Notably, this project identified one class of relaxed groups and two classes of unrelaxed groups. Class III relaxed galaxy groups contain one BGG that is notably brighter than surrounding members (Shen et al. 2014). I identified two classes of unrelaxed groups in all richness categories. Class I unrelaxed galaxy groups have two or three members with comparable luminosities that are larger than the luminosities of other group members. All member galaxy luminosities are comparable in Class II groups. Both trends seem independent of the magnitude gap and known sample biases. Trends characterized by the Class I and Class II designations of dynamically active groups may be related to evolutionary mechanisms that act on satellite galaxies. I do not explore satellite galaxy interactions in my BGG-focused analysis properly. Further analysis of member velocity dispersions and positional data and comparison to group formation simulations may aid in determining the physical meaning behind the unrelaxed, intermediate, and relaxed galaxy group classes.

Figures 1- 7, 9, 11: Color-Magnitude-Position Diagrams (Selection)

Member galaxies (o) plotted for their $\triangle \mathrm{RA}$ or $\triangle \mathrm{DEC}$ positions defined in Fosdick 2020 around the group's center (x). Each marker is scaled for the member galaxy's r-band absolute magnitude as described in Methods. Marker color corresponds to the member galaxy's g-r color. Legends are made transparent when they obscure figure data. 
Tempel et al. 2014 Group 39: $\triangle R A$ vs. $\triangle D E C$ with Color and Abs. Magnitude

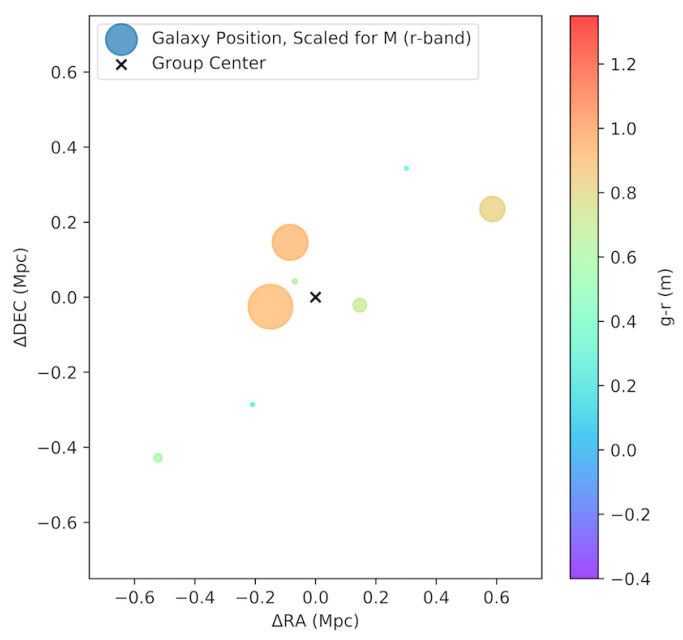

Berlind et al. 2006 Group 24: $\triangle$ RA vs. $\triangle \mathrm{DEC}$ with Color and Abs. Magnitude

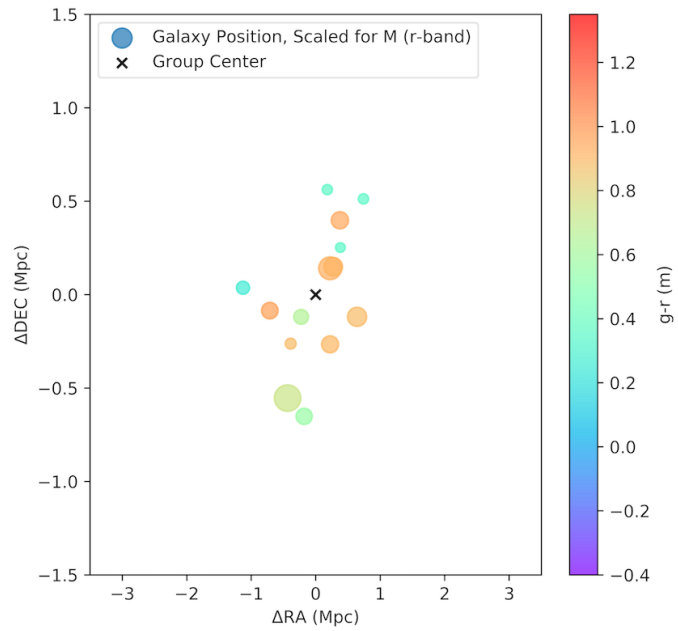

Berlind et al. 2006 Group 3896: $\triangle R A$ vs. $\triangle D E C$ with Color and Abs. Magnitude

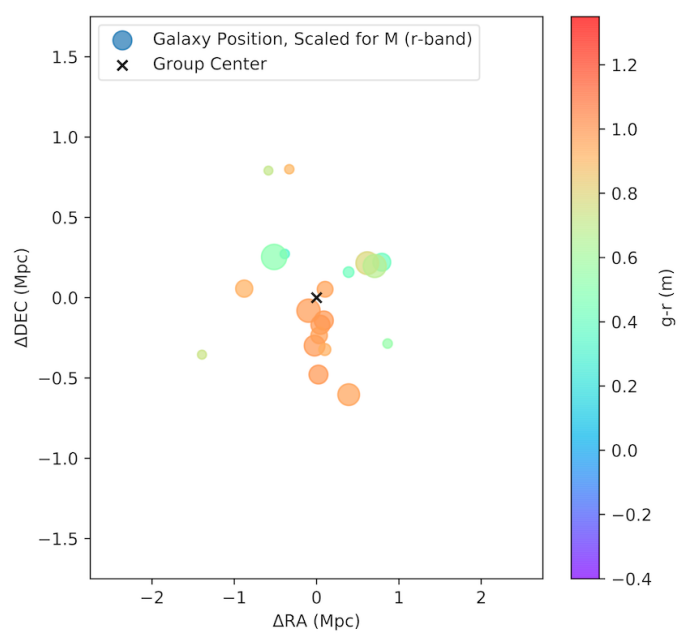

Berlind et al. 2006 Group 82: $\triangle R A$ vs, $\triangle D E C$ with Color and Abs, Magnitude

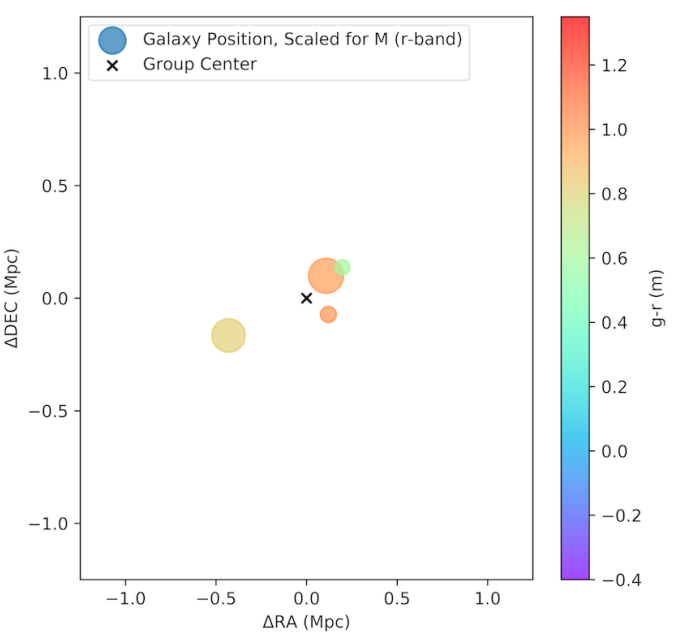

Tempel et al. 2014 Group 24: $\triangle R A$ vs. $\triangle D E C$ with Color and Abs. Magnitude

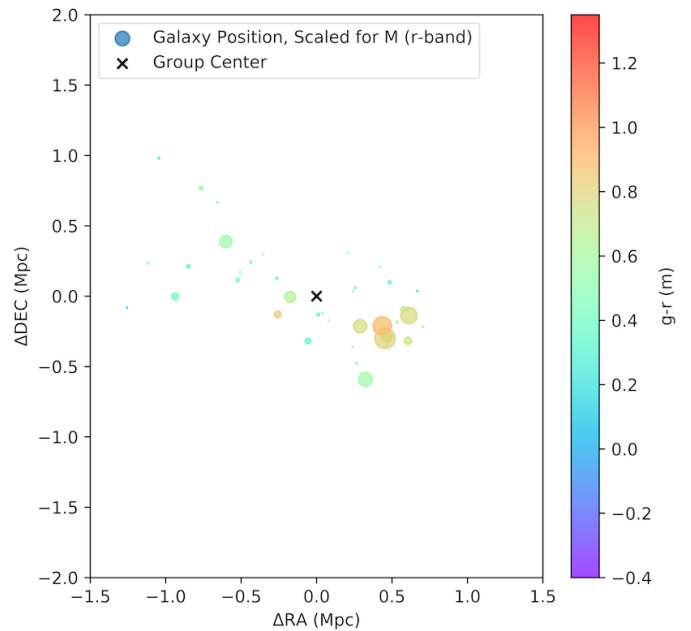

Berlind et al. 2006 Group 3064: $\triangle$ RA vs. $\triangle D E C$ with Color and Abs. Magnitude

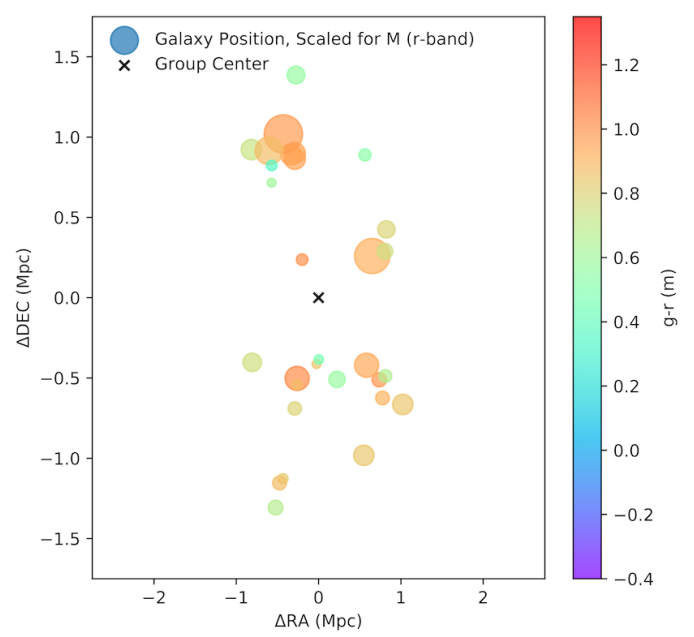


Tempel et al. 2014 Group 115: $\triangle \mathrm{RA}$ vs. $\triangle \mathrm{DEC}$ with Color and Abs. Magnitude

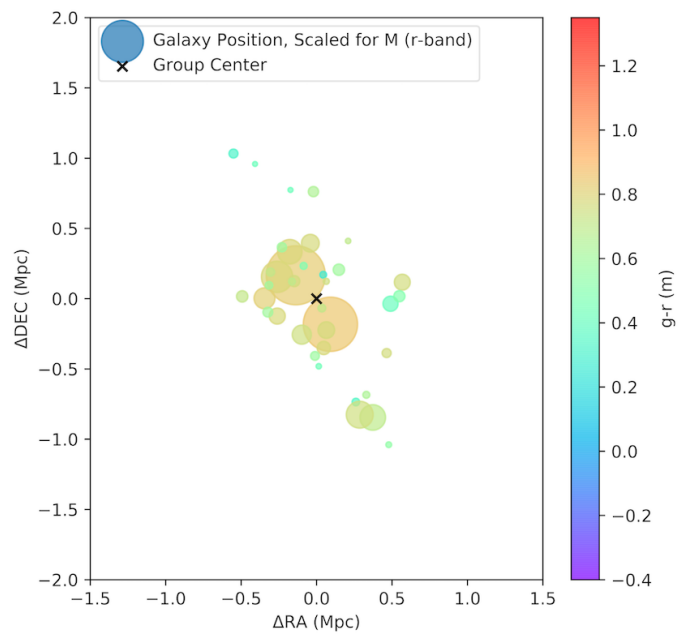

Berlind et al. 2006 Group 8245: $\triangle R A$ vs. $\triangle D E C$ with Color and Abs. Magnitude

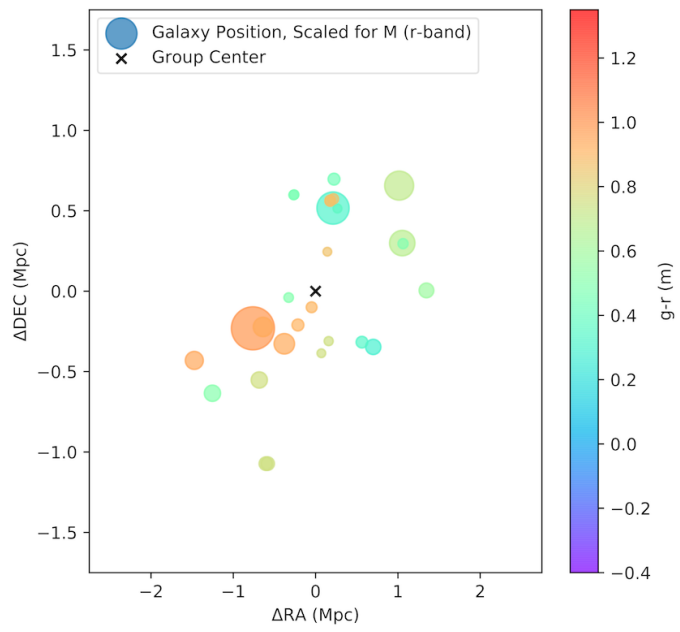

Tempel et al. 2014 Group 224: $\triangle$ RA vs. $\triangle \mathrm{DEC}$ with Color and Abs. Magnitude

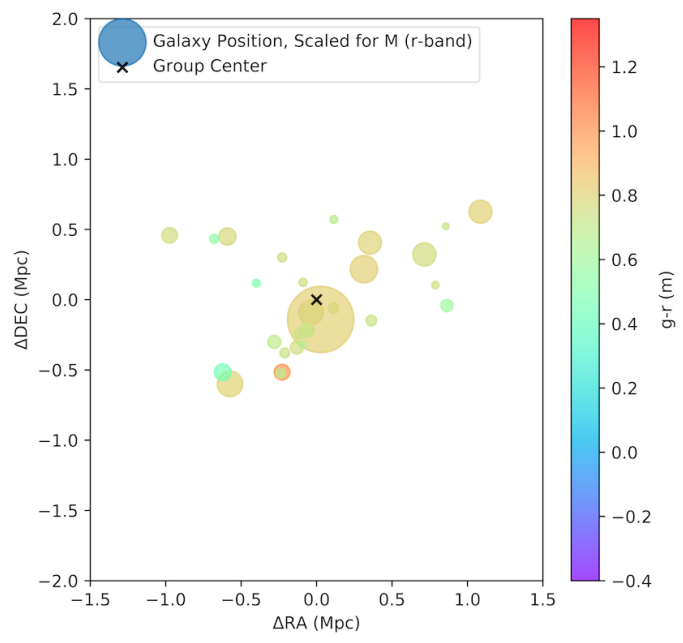

Tempel et al. 2014 Group 115: $\triangle R A$ vs. $\triangle D E C$ with Color and Stellar Mass

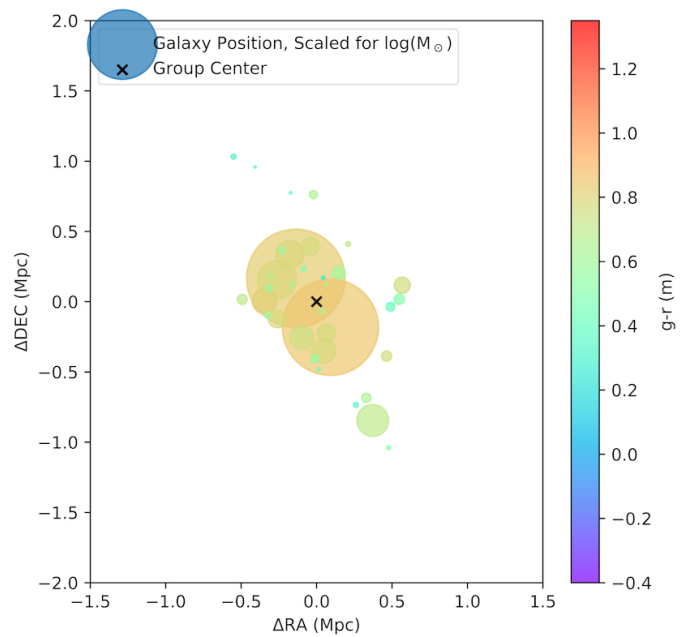

Berlind et al. 2006 Group 8245: $\triangle R A$ vs. $\triangle D E C$ with Color and Stellar Mass

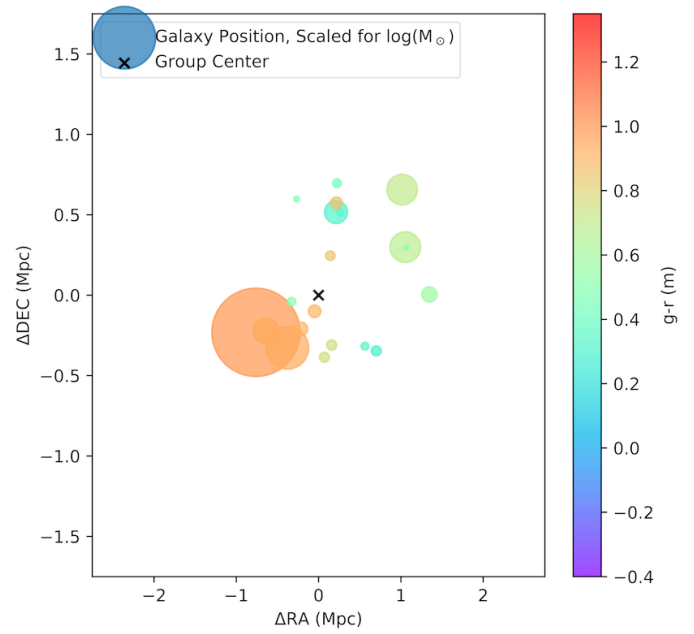

Tempel et al. 2014 Group 224: $\triangle R A$ vs. $\triangle D E C$ with Color and Stellar Mass

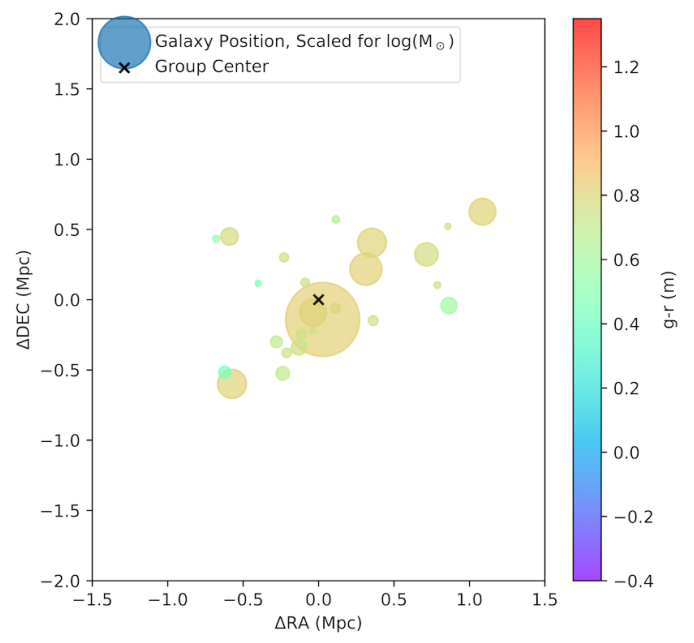


Figures 8, 10, 12: Color-Stellar Mass-Position Diagrams (Selection)

Member galaxies (o) plotted for their $\triangle \mathrm{RA}$ or $\triangle \mathrm{DEC}$ positions defined in Fosdick 2020 around the group's center (x). Each marker is scaled for the member galaxy's logarithmic stellar mass as described in Methods. Marker color corresponds to the member galaxy's g-r color. Legends are made transparent when they obscure figure data.

\section{ACKNOWLEDGEMENTS:}

This project would not be possible without generous funding from the Wisconsin Space Grant Consortium (WSGC) and National Space Grant College and Fellowship Program. I would also like to thank my research advisor Eric Wilcots for his continued support and guidance. I am grateful for the efforts of scientists at SDSS, the Berlind et al. 2006 group, and Tempel et al. 2014 group that provided data used during this project.

\section{REFERENCES:}

Berlind, A. et al. (2006). Percolation Galaxy Groups and Clusters in the SDSS Redshift Survey: Identification, Catalogs, and the Multiplicity Function. The Astrophysical Journal Supplement Series , 167(1), 1-25. doi: 10.1086/508170

Cooray, A. (2005). A divided Universe: red and blue galaxies and their preferred environments. Monthly Notices of the Royal Astronomical Society, 363(1), 337-252. doi: 10.1111/j.1365-2966.2005.09457.x

Conroy, C., Gunn, J. E., \& White, M. (2009). The Propagation Of Uncertainties In Stellar Population Synthesis Modeling. I. The Relevance Of Uncertain Aspects Of Stellar Evolution And The Initial Mass Function To The Derived Physical Properties Of Galaxies. The Astrophysical Journal, 699(1), 486-506. doi:10.1088/0004-637x/699/1/486

Dariush, A. A. et al. (2010). The mass assembly of galaxy groups and the evolution of the magnitude gap. Monthly Notices of the Royal Astronomical Society, 405(3), 1873-1887. doi: 10.1111/j.1365-2966.2010.16569.x

Dressler, A. (1980). Galaxy morphology in rich clusters - Implications for the formation and evolution of galaxies. The Astrophysical Journal, 236, 351-365. doi:10.1086/157753

Dressler, A. (1984). The Evolution of Galaxies in Clusters. Annual Review of Astronomy and Astrophysics, 22, $185-222$. doi:10.1146/annurev.aa.22.090184.001153

Eke, V. R., et al. (2004). Galaxy groups in the 2dFGRS: The group-finding algorithm and the 2PIGG catalogue. Monthly Notices of the Royal Astronomical Society, 348(3), 866-878. doi:10.1111/j.1365-2966.2004.07408.x

Freeland, E., Stilp, A., \& Wilcots, E. (2009). H I Observations of Five Groups of Galaxies. The Astronomical Journal , 138(1), 295-304. doi: 10.1088/0004-6256/138/1/295

Fosdick, K. C. (2020). The Evolution and Growth of Nearby Galaxy Groups. Proceedings of the 29th Annual Wisconsin Space Conference. doi: https://doi.org/10.17307/wsc.v1i1.282

Geller, M. J., \& Huchra, J. P. (1983). Groups of galaxies. III - The CfA survey. The Astrophysical Journal Supplement Series, 52 , 61-87. doi:10.1086/190859

Helsdon, S. F., \& Ponman, T. J. (2003). X-ray bright groups and their galaxies. Monthly Notices of the Royal Astronomical Society, 340(2), 485-498. doi:10.1046/j.1365-8711.2003.06320.x

Just, D. W. et al. (2010). The Environmental Dependence Of The Evolving S0 Fraction. The Astrophysical Journal, $711(1), 192-$ 200. doi:10.1088/0004-637x/711/1/192

Luparello, H. E., Lares, M., Paz, D., Yaryura, C. Y., Lambas, D. G., \& Padilla, N. (2015). Brightest group galaxies and the largescale environment. Monthly Notices of the Royal Astronomical Society, 448(2), 1483-1493. doi:10.1093/mnras/stv082 
Lintott, C. et al. (2010). Galaxy Zoo 1: Data release of morphological classifications for nearly 900,000 galaxies. Monthly Notices of the Royal Astronomical Society, 410(1), 166-178. doi:10.1111/j.1365-2966.2010.17432.x

Mahtessian, A. P., Movsisyan, V. H., Mahtessian, L. A., \& Karapetian, G. S. (2017). Dependencies between the magnitude gap of groups and the morphology of two brightest galaxies. CoBAO, 65(2), 401-406.

Raouf, M. et al. (2019). The Impact of the Dynamical State of Galaxy Groups on the Stellar Populations of Central Galaxies. The Astrophysical Journal, 887(2), 264. doi:10.3847/1538-4357/ab5581

Shen, S. et al. (2014). The Statistical Nature Of The Brightest Group Galaxies. The Astrophysical Journal, $782(1), 23$. doi:10.1088/0004-637x/782/1/23

Tago, E. et al. (2008). Groups of galaxies in the SDSS Data Release 5. Astronomy \& Astrophysics, 479(3), 927-937. doi:10.1051/0004-6361:20078036

Tempel, E. et al. (2011). Galaxy morphology, luminosity, and environment in the SDSS DR7. Astronomy \& Astrophysics, 529. doi:10.1051/0004-6361/201016196

Tempel, E. et al.(2014). Flux- and volume-limited groups/clusters for the SDSS galaxies: Catalogues and mass estimation. Astronomy \& Astrophysics, 566. doi:10.1051/0004-6361/201423585

Tempel, E., Tuvikene, T., Kipper, R., \& Libeskind, N. I. (2017). Merging groups and clusters of galaxies from the SDSS data. Astronomy \& Astrophysics (A\&A), 602. doi: 10.1051/0004-6361/201730499

Tully, R. B. (1987). Nearby groups of galaxies. II- an all-sky survey within 3000 kilometers per second. The Astrophysical Journal, 321, 280. doi:10.1086/165629

White, S. D., \& Rees, M. J. (1978). Core condensation in heavy halos: A two-stage theory for galaxy formation and clustering. Monthly Notices of the Royal Astronomical Society, 183(3), 341-358. doi:10.1093/mnras/183.3.341

Yang, X. et al. (2007). Galaxy Groups in the SDSS DR4. I. The Catalog and Basic Properties. The Astrophysical Journal, 671(1), 153-170. doi:10.1086/522027 